\title{
Music Therapy or Music Medicine?
}

\section{P.Barathi *}

The choice ultimately rests on the availability of skilled personnel as well as patient-preferred musical pieces, ease of administration and other factors......

An overwhelming increase in stress-induced psychosomatic ailments are evident today as a result of the impact of global crises culminating in social and environmental issues such as inflation, war, social violence, communicable diseases, epidemic outbreaks, etc., The ramifications are far-reaching, affecting not only the directly impacted victims and survivors, but also the indirectly affected among the population, such as those edged on the poverty line, middleclass people, and the class of business executives who strive to keep their endeavours going in spite of the vicious cycle of corruption, lay-offs, strikes and other non-cooperative movements by the workers.

Music therapy nourishes the mind and spirit of the psychologically afflicted individuals, but in a country like India it is not easily accessible to one and all, since it necessitates the need for trained personnel in order to establish a therapeutic relationship through music, involving the triad of music, client, and therapist. A trained music therapist implements a music intervention in an on-going therapeutic process, invoking personally tailored music experiences. The discipline of music therapy is still in its sprouting stage in the fertile soil of India, though it is exhibiting signs of rapid evolution from its nascent stage before a decade. Music medicine or the use of pre-recorded music comes to the rescue, since it is affordable, patient-friendly, with the possibility of the musical piece for selection by the patient himself for regular listening at home, under proper clinical supervision, and at suitable intervals..

\section{Elements and Relationships in Music Medicine}

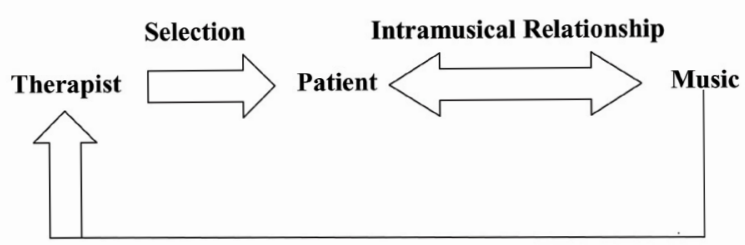

Music medicine is widely used in medical interventions to enhance or facilitate the treatment, and to assist rehabilitation. Music is used to influence the patients' physical, mental, or emotional states before, during, or after medical, dental, or paramedical treatments like physiotherapeutic procedures ( Bruscia,1998 ; Dileo,1999). There is usually no therapeutic relationship established through music administered during such procedures. From a paradigmatic point of view, music medicine might be seen as a cognitive-behaviourally oriented intervention model, since it is based on a stimulus-response paradigm, unlike music therapy which is purely relational. In music medicine interventions, the patient simply listens to pre-recorded music that is offered by a medical professional. There is evidence that both music medicine and music therapy are effective. Dileo and Bradt (2005) examined the therapeutic effects of the two practices in 11 medical areas, based on 183 controlled studies. Their conclusions were that both practices have a significant effect in 10 of the 11 areas, Therefore, it can be concluded that both music medicine and music therapy are effective and can serve as complementary practices in somatic care.

Now let us glean an insight into the discipline of music medicine from the perspective of a Cognitive-Behaviourally oriented intervention model.

In Cognitive-Behavioural Music Therapy (CBMT) music is used to modify behavior through conditioning, and

\footnotetext{
* Dr. P.Barathi, M.D.,

Prof of Physiology, Music Therapist

e-mail : iyer.bharthi@gmail.com
} 
the results can be analysed by applied behaviour analysis.

The therapeutic process is based on a stimulus- response paradigm, and as a dependent variable the music must be controlled. This explains why specific music, often recorded, is preferred to improvisation in the treatment of patients using this procedure (GroTrondalen and Ole Bonde,2012). Many types of behaviours such as physiological, motor, psychological, emotional, cognitive, perceptual, and autonomic behaviours are manipulated in CBMT. A patientpreferred music stimulus is given or withdrawn as related to the patient's target behavior, for example, continuing the music if the subject attentively listens to it, and vice versa. The session format has a firm structure as well as well-defined protocols, though it is adapted to suit the cognitive or behaviour modification goals. The sessions necessitate factors such as predictability and control of the musical stimulus. The music acts as a reinforcing stimulus of non-musical behavior$\mathrm{CBMT}$ is therefore music in therapy, music being used as a means to achieve cognitive and behavioural changes.

Neurological rehabilitation is an important arena for CBMT, and Michael Thaut has developed a specific training in 'neurological music therapy', based on the principles of CBMT ( Thaut, 2000, 2005). People with Parkinson's disease, autistic children, premature infants, geriatric population and adolescents with developmental disabilities respond to this therapy. CBMT is recognized by the scientific community, since it ensures the possibilities of replication and standardization. Extensive research has been carried out to determine what types of music will promote specific therapeutic and treatment objectives. Thaut has shown that pulsed, rhythmical music can facilitate good walking patterns in patients with Parkinson's disease. According to Thaut, the rhythm in itself has a high potential for gait training, and a metronome can be sufficient to produce the right stimulation ( Thaut and Abiru, 2010). Music with slower tempos is used when older adults are prompted to move or dance. The effect of the music medicine/ music therapy intervention over time is assessed through applied behavior analysis by the researcher or the therapist.

According to a Cochrane systematic review headed by Dr. Joke Bradt of Drexel University, Philadelphia,USA, both music therapy and music medicine employing pre-recorded music demonstrate positive results, and that there is not enough evidence currently to determine if one intervention is more effective than the other. The trials were conducted with cancer patients that had been offered recorded music, (17 patients) or music therapy with a trained therapist( 13 patients). The results indicate that both music medicine and music therapy remarkably decrease anxiety based on clinical anxiety scores in comparison to those on standard treatment regimes. Similar studies done on patients subjected to mechanical ventilation, and coronary heart disease reveal that both types of music interventions are effective in improving psychological and physical outcomes.

My opinion is that in addition to conventional therapies, clinicians should also focus on developing suitable music medicine protocols (apart from nurses and music psychologists), in order to extend soothing hands to embrace the stress-afflicted population, loaded inadvertently with toxic allopathic drugs which leave their undesirable, permanent imprints on liver and renal physiology.

\section{REFERENCES}

1.Bruscia, K.E.(1998). Defining music Therapy. Second Edition ed. Lower Village: Barcelona Publishers.

2.Dileo, C.(ed.) (1999). Music Therapy and Medicine: Clinical and Theoretical Applications. Silver Spring, MD: American Music Therapy Association.

3.Dileo, C. and Bradt, J.(2005). Medical Music Therapy: A Meta-Analysis of the literature and an Agenda for Future Research Cherry Hill, NJ: Jeffrey Books.

4.GroTrondalen and Lars Ole Bonde (2012). Music Therapy: Models and Interventions : Music, Health and WellBeing. Eds Raymond Mac Donald, Gunter Kreutz, and Laura Mitchell, Oxford University Press.

5.Thaut, M.H.(2000). A scientific model of music in therapy and medicine.St. Louis, MO: MMB Music.

6.Thaut, M.H.(2005). Rhythm, human temporality, and brain function. In : D. Miell, R. Mac Donald, and D. J. Hargreaves(eds.) Musical Communication, pp.171-92. Oxford: Oxford University Press.

7.Thaut, M. and Abiru, M.(2010). Rhythmic auditory stimulation in rehabilitationof movement disorders: A review of current research. Music Perception: An Interdisciplinary Journal, 27(4), 263.

8.Bradt,J., Dileo,C. Grocke,D., Magill, L.(2011). Music interventions for improving psychological and physical outcomes in cancer patients. Cochrane Database of Systematic Reviews, issue 8. Art. No: CD006911 